\section{Heterojunction Solar Cell Fabricated with Single- Crystalline GaN Nanorod Array}

Arrays of one-dimensional nanostructures have important potential as building blocks for a variety of optoelectronic devices. They may be particularly useful in solar cells, because of their ability to carry photogenerated charges away from a junction region, thus minimizing recombination rates and improving conversion efficiencies. Now a team of researchers led by C.S. Lee and S.T. Lee at the City University of Hong Kong, H.T. Cong at the Chinese Academy of Sciences, and their collaborators have demonstrated a heterojunction solar cell based on an array of $p$-type GaN nanorods on an $n$-type Si substrate, with promising photovoltaic properties. They reported their findings in the December 10, 2008 issue of Nano Letters (DOI: 10.1021/nl801728d; p. 4191).

Gallium nitride is appealing for use in nanodevices because of its wide, direct bandgap, high-carrier mobility, good thermal and chemical stability, and its ability to be $p$ - or $n$-doped. GaN nanorods are relatively easily grown on Si substrates with an oriented morphology and a large bandgap difference, making this an attractive potential combination for photovoltaic applications. Motivated by this logic, the Hong Kong group synthesized $\mathrm{Mg}$-doped $\mathrm{GaN}$ nanorod arrays by thermally evaporating a powder mixture of $\mathrm{GaCl}_{3}$ and $\mathrm{MgCl}_{2}$ (molar ratio 30:1) for one hour at $850^{\circ} \mathrm{C}$ in a flow of high-purity ammonia and hydrogen onto a Si substrate that had been seeded with gold nanoparticles. The resulting nanorods had a uniform size distribution of approximately $100 \mathrm{~nm}$ in diameter and $1.0 \mu \mathrm{m}$ in length, and were confirmed by high-resolution transmission electron microscope images to have a single-crystal wurtzite structure with no observable defects or amorphous shells. Energy-dispersive x-ray spectroscopy revealed that the $\mathrm{Mg}$ was uniformly distributed in each nanorod, in concentrations varying from 1.1 to 2.4 at. $\%$.

The researchers next fabricated a solar cell using one of the arrays, by filling the spaces between the nanorods with insulating photoresist (PMMA) and then electron-beam evaporating a $\mathrm{Ni} / \mathrm{Au}$ (30/50 nm thicknesses) electrode onto the nanorods and a $\mathrm{Ti} / \mathrm{Al}$ (30/50 nm thicknesses) electrode onto the backside of the Si substrate. This $p$-GaN nanorod $/ n-\mathrm{Si}$ heterojunction device, measuring $0.5 \times 0.5 \mathrm{~cm}^{2}$, was exposed to AM1.5G solar illumination with an intensity of $100 \mathrm{~mW} / \mathrm{cm}^{2}$, and was found to have a rectification ratio of better than $10^{4}$ at $\pm 0.5 \mathrm{~V}$, a fill factor of 0.38 , and a power conversion efficiency of $2.73 \%$. Additionally, the nanorod array displayed a relatively low reflection coefficient, as was expected from its one-dimensional array form, thus functioning as an antireflection coating for the device.

The researchers said that these "aligned GaN nanorods can be directly incorporated into the device structure without a complicated fabrication process." These results suggest that one-dimensional nanostructure arrays of GaN are promising components in heterojunction solar cells, and may one day lead to a technology impact rivaling that of $\mathrm{GaN}$ in light-emitting diodes.

COLIN MCCORMICK

\section{Urease-Functionalized Silica Enables Self-Mineralization}

Hydroxylapatite (HAp) is found in teeth and bones, and is commonly used as a filler or surface coating in bone repair. Typically, coating deposition requires energetic, physically driven processes, such as plasma spraying and magnetron sputtering. Even solution-based processes require hydrothermal conditions that make unviable incorporation of labile bioactive compounds. Alternative, soft chemistry techniques require long time periods for HAp-like film formation. Biologically driven mineralization processes, however, have inspired biomimetic approaches for the preparation of biocomposites under mild conditions. Recently,

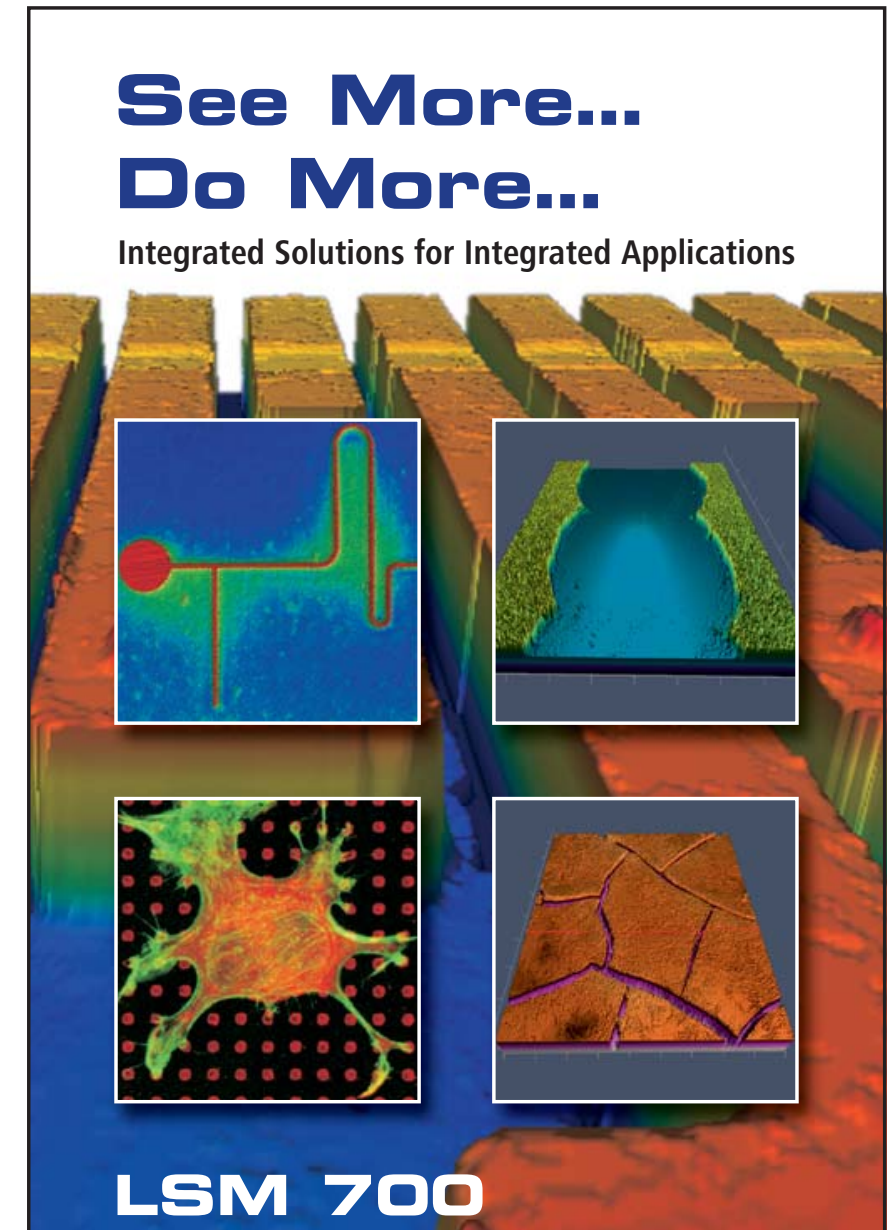

\section{Confocal Laser Scanning Microscope}

Insight into Materials Science through:

- 3D Imaging

- Surface topography / Roughness

- Volumetric measurement

- Multi-label Imaging

- Fluorescence \& Reflected light imaging

Applications in Research \& Quality Assurance:

- Microfluidics - Sensors

- Microarrays - Lab-on-Chip

- Microreplication

- Bio Materials

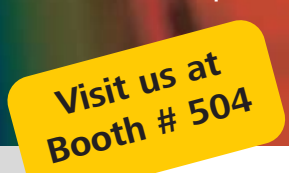

Carl Zeiss Microlmaging, Inc.

One Zeiss Drive

Thornwood, NY 10594

1-800-233-2343

micro@zeiss.com

www.zeiss.com/materials

Register to win a

pair of ZEISS binoculars

www.zeiss.com/binocs 3

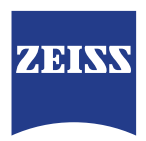

We make it visible 
M. Jobbágy and co-researchers at Consejo Superior de Investigaciones CientíficasCSIC, Madrid, Spain, Universidad de Buenos Aires, Argentina, and Centro Interdisciplinario de Nanociencia y Nanotecnologia, used urease-functionalized silica as an interface for nucleation and growth of HAp-like coatings under mild conditions and short aging times.

As reported in the December 23, 2008 issue of Chemistry of Materials (DOI: 10.1021/cm8021566; p. 7368), Jobbágy and co-researchers functionalized monodisperse, submicrometer silica spheres as model substrates by covalently attaching urease. The bioactive silica was then aged for 6 hours in a mineralizing solution containing $\mathrm{Ca}(\mathrm{II}), \mathrm{P}(\mathrm{V})$, and urea, at $\mathrm{pH} 4.0$ in order to maximize $\mathrm{Ca}(\mathrm{II})$ and $\mathrm{P}(\mathrm{V})$ solubility and prevent irreversible urease denaturation. A control experiment was performed with a nonsilica substrate. Transmission electron microscopy (TEM) showed that mineralization transformed the smooth surfaces of the urease-capped silica spheres to rough surfaces with flakelike texture, while the control spheres remained smooth. The researchers observed no uncoated or partially coated silica spheres, nor did they observe free HAp-like flakes greater than $10 \mathrm{~nm}$ in length. The mineral coating was characterized with TEM and scanning electron microscopy (SEM) in conjunction with energy dispersive spectroscopy (EDS). TEM-EDS showed that the coating's Ca/P ratio for isolated spheres is about 1.6, which is close to that for HAp, while SEMEDS showed the same composition for large clusters of coated spheres. X-ray diffraction confirmed the coating's crystalline nature. The researchers said that the mechanism of coating formation is likely direct growth of HAp-like particles onto the silica surface rather than homogeneous nucleation followed by heterocoagulation of HAp-like particles onto silica. The researchers also said, "This method can be applied over different geometries, allowing the design of novel biocompatible films, scaffolds, core-shell nanoparticles, and so forth. Since other relevant carbonate- and/or fluoride-based biominerals can also be developed by urease, in the presence of labile macromolecules or gels, this route allows the preparation of a wide range of biocomposites."

STEVEN TrohalaKI

\section{Accordion-Like Honeycombs Achieve Compatibility for Cardiac Tissue Engineering}

The fundamental goal of tissue engineering is to make tissue-engineered constructs with a potential to replace the biological functions of damaged organs. There is now worldwide activity in the in vitro regeneration of tissues including skin, nerve, liver, cartilage, bone, heart valves, blood vessels, and kidney. For myocardial tissue engineering, the key is to engineer three-dimensional (3D) cardiac tissue that could eventually be used to repair damaged heart tissue inside the body, test new cardiac drugs, and study cardiac cell development and functions. In principle, it could theoretically lead to the creation of an entire heart. Among the major challenges in developing tissue-engineered grafts for myocardial repair are to achieve structural and mechanical compatibility of 3D scaffolds with the formation of new biomimetic tissue. G.C. Engelmayr Jr. of the Massachusetts Institute of Technology (MIT), L.E. Freed and C.J. Bettinger of MIT and the Charles Stark Draper Laboratory, and their colleagues have recently attempted to overcome this challenge by

\section{High Resolution}

National Electrostatics Corporation has added Ångstrom level, High Resolution RBS to the RC43 Analysis System for nanotechnology applications. A single Pelletron instrument can now provide RBS, channeling RBS, microRBS, PIXE, ERDA, NRA, and HR-RBS capability, collecting up to four spectra simultaneously. Pelletron accelerators are available with ion beam energies from below $1 \mathrm{MeV}$ in to the $100 \mathrm{MeV}$ region.

www.pelletron.com

Phone: 608-831-7600

E-mail: nec@pelletron.com

National Electrostatics Cor $p_{y}$ 